\title{
The lateral extracavitary approach to the thoracolumbar spine: a case series and systematic review
}

\author{
Paul M. Foreman, MD, ${ }^{1}$ Robert P. Naftel, MD, ${ }^{2}$ Thomas A. Moore II, MD, ${ }^{3}$ and Mark N. Hadley, MD ${ }^{1}$ \\ Departments of ${ }^{1}$ Neurological Surgery and ${ }^{3}$ Anesthesia, University of Alabama at Birmingham, Alabama; and ${ }^{2}$ Department of \\ Neurological Surgery, Vanderbilt University, Nashville, Tennessee
}

\begin{abstract}
OBJECT Since its introduction in 1976, the lateral extracavitary approach (LECA) has been used to access ventral and ventrolateral pathology affecting the thoracolumbar spine. Reporting of outcomes and complications has been inconsistent. A case series and systematic review are presented to summarize the available data.
\end{abstract}

METHODS A retrospective review of medical records was performed, which identified 65 consecutive patients who underwent LECA for the treatment of thoracolumbar spine and spinal cord pathology. Cases were divided according to the presenting pathology. Neurological outcomes and complications were detailed. In addition, a systematic review of outcomes and complications in patients treated with the LECA as reported in the literature was completed.

RESULTS Sixty-five patients underwent the LECA to the spine for the treatment of thoracic spine and spinal cord pathology. The most common indication for surgery was thoracic disc herniation (23/65, 35.4\%). Neurological outcomes were excellent: $69.2 \%$ improved, $29.2 \%$ experienced no change, and $1.5 \%$ were worse. Two patients (3.1\%) experienced a complication. The systematic review revealed comparable neurological outcomes $(74.9 \%$ improved) but a notably higher complication rate $(32.2 \%)$.

CONCLUSIONS The LECA provides dorsal and unilateral ventrolateral access to and exposure of the thoracolumbar spine and spinal cord while allowing for posterior instrumentation through the same incision. Although excellent neurological results can be expected, the risk of pulmonary complications should be considered.

http://thejns.org/doi/abs/10.3171/2015.6.SPINE15169

KEY WORDS lateral extracavitary approach; thoracic spine; thoracic spinal cord; complication; outcome

$\mathrm{T}$

ThE lateral extracavitary approach (LECA) to the thoracolumbar spine was originally described by Larson et al. in 1976 for the treatment of traumatic pathology. ${ }^{16}$ This approach provides dorsal and ventrolateral access to the thoracolumbar spine without the morbidity associated with traditional transpleural and transperitoneal approaches. Additionally, it allows for posterior instrumentation through the same surgical incision. Since its description in 1976, the popularity of the approach has grown, and indications for the LECA have expanded to include disc herniation, infection, tumor, and deformity.

The current literature on the LECA varies widely in the reporting of surgical indications, outcomes, and complications. A case series and systematic review of the literature are presented herein, detailing outcomes and complications accompanying the LECA to the thoracolumbar spine in an effort to synthesize the available data.

\section{Methods \\ Case Series \\ Patient Selection}

Following institutional review board approval from the University of Alabama at Birmingham, a database of all patients who underwent a lateral extracavitary approach to the thoracolumbar spine was retrospectively populated from medical records. Sixty-five consecutive patients who underwent this approach for any pathology at the University of Alabama at Birmingham Hospital by the senior author (M.N.H.) between 1993 and 2011 were included.

Patients were categorized by their indication for surgery. Surgical outcomes were evaluated separately in patients with nontraumatic indications and traumatic indications for surgery. Length of follow-up was calculated using the length of time from the date of surgery to the most

ABBREVIATIONS ASIA = American Spinal Injury Association; DVT = deep venous thrombosis; JOA = Japanese Orthopaedic Association; LECA = lateral extracavitary approach.

SUBMITTED February 5, 2015. ACCEPTED June 30, 2015.

INCLUDE WHEN CITING Published online December 18, 2015; DOI: 10.3171/2015.6.SPINE15169. 
recent clinic or hospital visit. Outcomes for patients undergoing surgery for nontraumatic indications were stratified as improved, no change, or worse. Outcomes for patients undergoing LECA surgery for traumatic indications (thoracic fractures, dislocations, with or without spinal cord injury) were defined using the American Spinal Injury Association (ASIA) Impairment Scale. ${ }^{10}$ These patients were stratified based on preoperative ASIA grade, and outcomes are presented as improved, no change, or worse based on the last follow-up ASIA grades.

\section{Operative Technique}

Prior to patient positioning, the operative table (Skytron 6500 elite, Skytron) is prepared for surgery. A bolster is inserted under a Wilson frame (Mizuho Orthopedics Systems, Inc.) on the side ipsilateral to the surgical pathology. The frame is taped securely to the table (Fig. 1). The table is tilted on its long axis to lower the side of the bed ipsilateral to the pathology, so that the patient's torso can initially be positioned parallel to the floor.

The patient is placed prone while under general anesthesia. A curvilinear skin incision is made beginning at the midline 3 spinal levels above the pathology and ending at the midline 3 spinal levels below the pathology. The apex of the arc of the incision is $7.5 \mathrm{~cm}$ off midline ipsilateral to the pathology (Fig. 2A). Intraoperative radiography or fluoroscopy is used to confirm the anatomical level. If the patient requires internal fixation and fusion in addition to thoracic spinal cord decompression, this incision accommodates the insertion of internal fixation screws, rods, and fusion substrate. After sterile preparation of the operative field, a skin incision is performed and dissection proceeds through the subcutaneous tissues to the level of the thoracodorsal fascia that overlies the latissimus dorsi and paraspinal musculature. This thick cutaneous flap is elevated, carried across the midline to the contralateral side, and reflected away from the surgeon (Fig. 2B). Next, the spinous processes and lamina are exposed through a subperiosteal dissection at the level above and below the pathology. For decompression-only cases, the dissection is only performed on the ipsilateral side. If stabilization and fusion are necessary, then bilateral exposure above and below the level of the pathology is required. The midline ipsilateral dissection proceeds laterally to identify the facet complex and rib head at the level of the pathology. The rib head belongs to the rib of the inferior level of the pa- thology (e.g., for T8-9 pathology, the T-9 rib head would be at the level of the pathology).

Next, a lateral dissection is conducted. The latissimus dorsi muscle is identified laterally at the apex of the skin incision, and a vertical incision is made in the latissimus fascia parallel to its muscle fibers down to the depth of the ribs. Dissection proceeds under the muscle along the dorsal side of the ribs, which allows mobilization of this ipsilateral latissimus muscle bundle. The mobile, but intact, latissimus and paraspinal muscle bundle can be reflected laterally when subperiosteal dissection is required medially, and then, reflected medially for rib resection. The lateral extracavitary approach is accomplished beneath this bundle from lateral to medial (Fig. 2C). The upper and midthoracic ribs articulate high on the lateral thoracic vertebrae; their superior-most portions are at the superior intervertebral disc space. The rib at the level of the pathology is dissected away from the intercostal muscles from lateral to medial. In general, care is taken to preserve the neurovascular bundle, but it may be sacrificed for additional exposure. This is common in cases of traumatic pathology and tumor, but is rarely needed for disc herniation. Next, the rib is stripped from the pleura, taking great care not to cause a pleural rent. The rib is then cut $6.0 \mathrm{~cm}$ from its head and removed.

With the latissimus muscle mobilized and the rib and rib-head resected, the surgeon can work beneath the latissimus muscle bundle to obtain exposure to the lateral and ventral aspect of the thoracic spinal column. Dissection medial to the muscle bundle allows the ipsilateral facet complex, the transverse process, and the proximal rib head to be resected at the desired level. At this point, the operating microscope is brought into the field for improved illumination and magnification. With the use of a high-speed drill, an ipsilateral hemilaminectomy is accomplished exposing the dorsal and lateral aspects of the thecal sac from the inferior aspect of the rostral pedicle to the superior aspect of the caudal pedicle (when treating pathology at 1 interspace level). By working under the lateral edge of the thecal sac, the disc space can be directly visualized (Fig. 2D).

When this operative approach is used to accomplish thoracic discectomy (Fig. 3), once the aforementioned dissection and lateral identification of the disc space has been accomplished, the surgeon works under the latissimus bundle, identifies the ventral aspect of the spinal canal (as

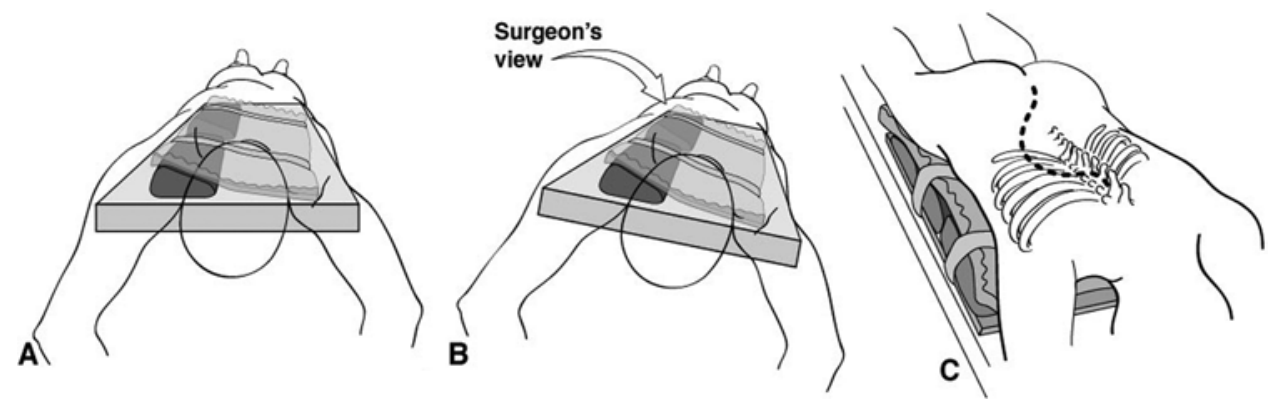

FIG. 1. A: Patient positioned prone on a Wilson frame atop the operative table with a bolster ipsilateral to the surgical pathology. B: After securing the patient to the operative table, the table itself can be rotated away from the surgeon, facilitating visualization ventral to the spinal column and across the midline. C: Surgical incision with respect to the spinal column and ribcage; note that the surgical incision is ipsilateral to the bolster and the surgical pathology. 

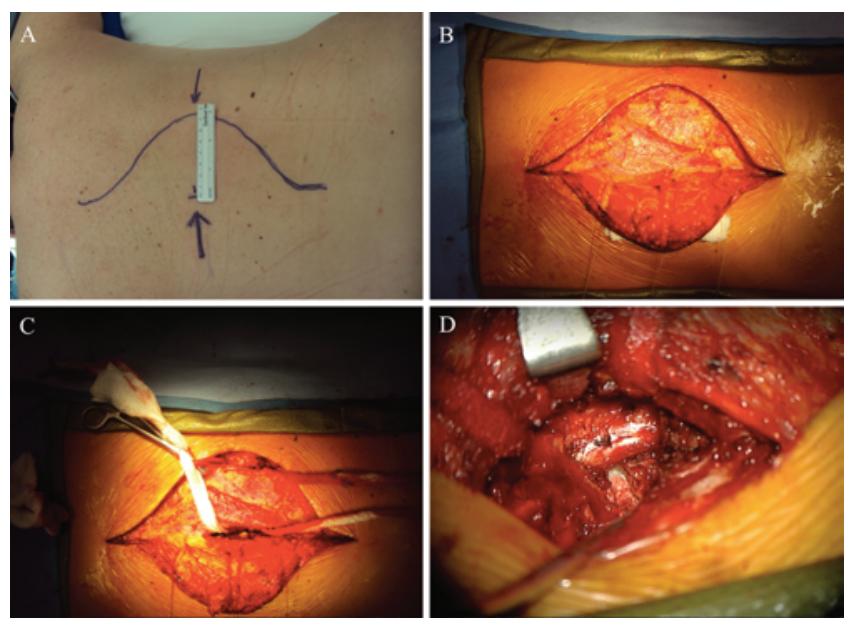

FIG. 2. A: Arc-type incision with the apex $7.5 \mathrm{~cm}$ off midline ipsilateral to the surgical pathology. B: A cutaneous flap is elevated, carried across the midline, and reflected away from the surgeon. C: Ipsilateral latissimus and paraspinal musculature are mobilized, allowing for both medial and lateral reflection of the intact muscle bundle. D: View from lateral demonstrating ventral access to the intervertebral disc space with direct visualization of the dorsal and lateral thecal sac. Figure is available in color online only.

defined by the lateral edge of the posterior longitudinal ligament), and drills away the inferior endplate and caudal $1.0 \mathrm{~cm}$ of the superior vertebral body and the superior endplate and rostral $1.0 \mathrm{~cm}$ of the inferior vertebral body. The surgeon works both above and below the disc space toward the ventral midline of the spinal canal, thus accomplishing partial unilateral vertebrectomy on each side of the disc space. The patient can be rotated away from the surgeon, facilitating bone dissection and operative exposure across the anatomical midline. Once this bone dissection has been accomplished, both above and below the intervertebral disc, down-pushing curettes may be used between the posterior longitudinal ligament and the thin remaining cortical bone of the ventral lateral canal to push the eggshell-thin cortical mantel into the bony dissection cavity. Finally, the remaining calcified shell of the ventral-lateral disc herniation may be forced downward into the dissection cavity, decompressing the cord from its ipsilateral lateral edge to just across the anatomical midline. Only the contralateral aspect of the thecal sac at this level cannot be directly visualized or decompressed via this exposure.

When this exposure is performed for intradural extramedullary pathology ventrolateral to the thoracic spinal cord, the exposed ventrolateral thecal sac may be opened laterally and retracted ventrally. A lateral dural opening of this type facilitates dural closure following tumor excision. With the patient rotated away from the surgeon, the lateral exposure allows the tumor to be microsurgically resected and forced into the bony vertebrectomy cavity and removed without manipulation or retraction of the spinal cord.

When this operative approach is used to treat trauma, infection, or other extradural pathology that extends beyond a single thoracic interspace, near-complete unilateral vertebrectomy can be accomplished via this exposure, at
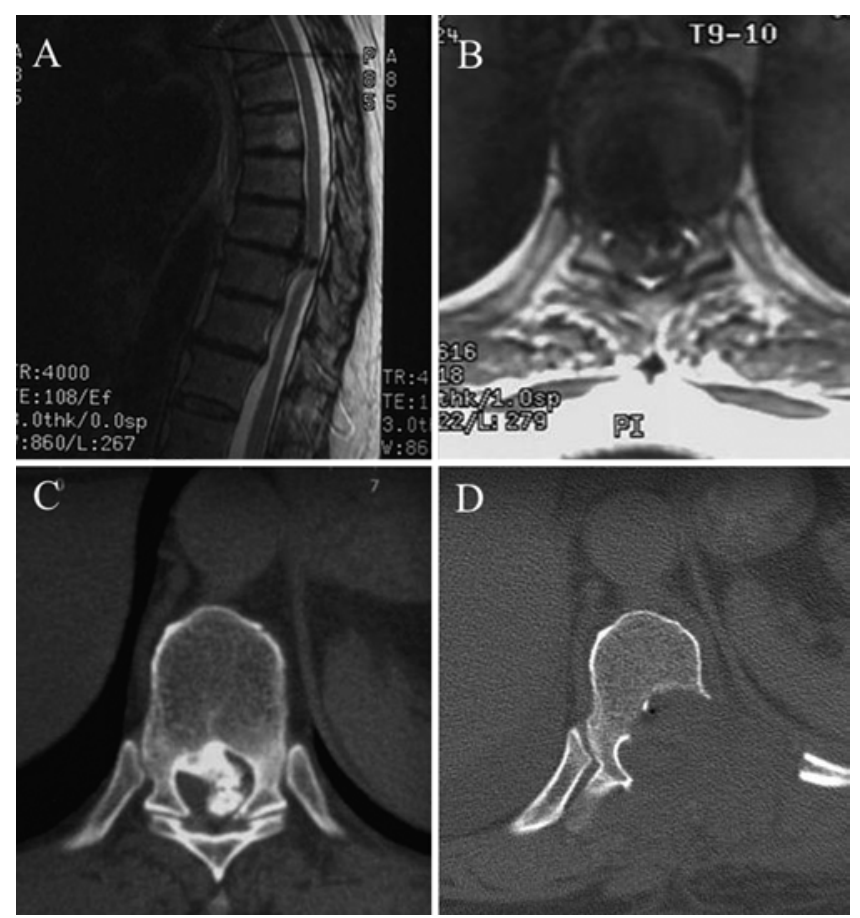

FIG. 3. Images obtained in a 68-year-old woman with thoracic pain and myelopathy. A: Preoperative sagittal MR image demonstrating T8-9 calcified disc herniation with spinal cord compression. B: Preoperative axial MR image demonstrating T8-9 calcified disc herniation that is eccentric to the left with compression and translation of the spinal cord to the right. C: Preoperative axial CT image demonstrating T8-9 calcified disc herniation that is eccentric to the left. D: Postoperative axial CT image following LECA for thoracic disc excision at T8-9.

multiple levels if necessary, to resect the involved vertebrae and to provide adequate exposure and decompression of the ipsilateral and ventral thecal sac and spinal cord. Intervertebral strut fusion, either with a bone strut or a bone-filled mechanical strut device, can be accomplished when the decompression has been completed. Ideally, the surgeon works between the thoracic nerve roots ipsilateral to the exposure. However, sacrifice of a unilateral thoracic nerve root may be required.

Once thoracic cord decompression has been accomplished hemostasis is obtained and the wound is irrigated with antibiotic solution. If internal fixation and fusion are to be part of the procedure (uncommon for discectomy or intradural tumor procedures, but common for trauma and/or vertebral body tumor resection procedures), anterolateral vertebral column reconstruction with an intervertebral strut graft is accomplished if necessary. Thoracic pedicle screws are placed bilaterally, typically 2 segments above and 2 segments below the level of pathological instability (Fig. 4). Autograft bone is placed bilaterally and dorsolaterally, completing the procedure.

The wound is closed over 2 surgical drains, one placed deep at the decompression site under the latissimus muscle bundle. The lateral opening of the latissimus dorsi fascia is closed. The medial portion of the paraspinous muscle/ latissimus dorsi muscle fascia is reattached to the interspinous fascia at the midline. The second Hemovac drain is placed over the muscle, under the subcutaneous flap. The 


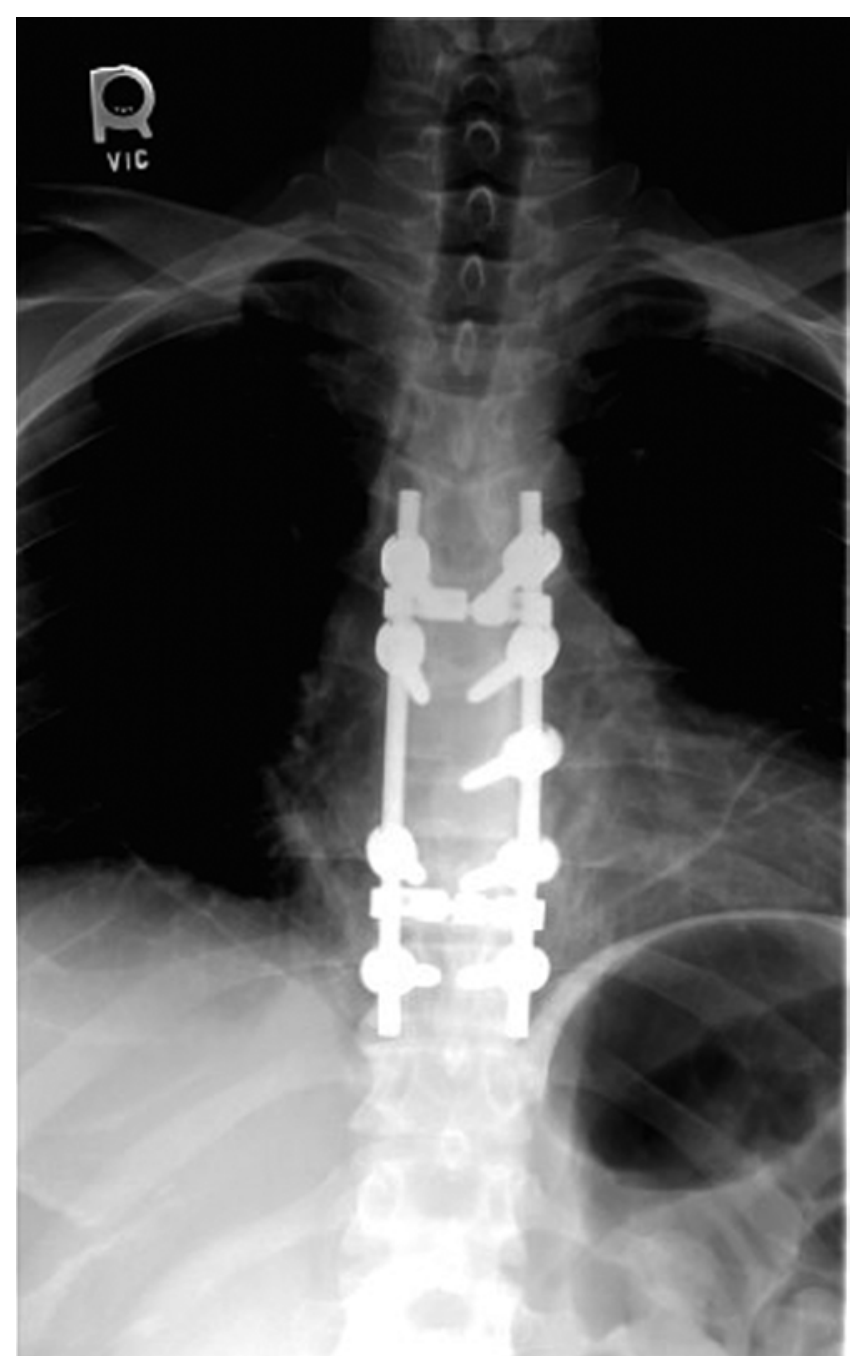

FIG. 4. Image from a 24-year-old man with ASIA Grade A spinal cord injury following a traumatic fracture dislocation. Postoperative radiograph of the thoracic spine following a right-sided LECA for a T6-10 internal fixation and fusion.

subcutaneous flap is moved back into appropriate juxtaposition and then closed in layers with both $2-0$ and 3-0 Vicryl sutures. The skin edges are approximated with a running nylon suture.

\section{Systematic Review}

A systematic review was conducted in accordance with the Preferred Reporting Items for Systematic Reviews and Meta-Analyses Statement. ${ }^{24}$ A PubMed literature search was performed November 14, 2014, to identify published peer-reviewed articles of interest. The database was searched using the key words "lateral extracavitary" to identify original case series of patients with thoracolumbar pathology treated with open LECA to the spine. Only clinical articles published in the English language were considered. Titles and abstracts of relevant articles were appraised in detail. The citations of relevant articles were reviewed and additional manuscripts included when appropriate. Pertinent full-text articles were then evaluated for inclusion in the LECA Summary Table (Table 1). The inclusion criteria were 1) age $\geq 16$ years, 2) thoracolumbar spine pathology, 3) utilization of open LEAC to the spine, 4) dataset of $\geq 5$ patients, and 5) report of complications or outcomes, or both of these. Eleven of the 63 articles (62 from the PubMed search, 1 from manuscript citations) were included in the LECA Summary Table (Table 1). ${ }^{1,4,8,9,14,16,19-22,26}$

The LECA Summary Table (Table 1) details the included studies. Study design, class of medical evidence, number of patients, levels of pathology, type of pathology, neurological outcome, pain outcome, and complications were included when mentioned in the original text. Neurological and pain outcomes were grouped into "improved," "no change," and "worse." Complications were grouped into "major," "minor," and "reoperation." Major complications were considered those that imparted permanent harm to the patient (i.e., spinal cord injury) and those that threatened mortality (i.e., sepsis, cecal volvulus). Minor complications included any untoward and reported event that did not meet criteria for a major complication (i.e., chest tube, pleural rent, CSF leak, wound infection, pneumonia, and deep venous thrombosis [DVT]). If a single event resulted in the reporting of more than 1 complication (i.e., a pleural rent resulting in a pneumothorax treated with a chest tube), it was considered 1 complication. A return to the operating room was considered a "reoperation" when it resulted directly from the original LECA operation (i.e., repositioning of spine hardware, repair of incisional hernia). Subsequent procedures not directly related to the original LECA operation (i.e., tracheostomy) were not included as reoperations. When a reoperation was known to be the result of either a major or minor complication, it was noted, to avoid counting a complication with reoperation as separate entities. The total number of patients suffering a complication was also included when mentioned in the original article.

\section{Results}

\section{Case Series}

\section{Baseline Characteristics}

Sixty-five patients underwent the LECA for a vertebral column and spinal cord pathology, ranging from the T-4 to the T-12 level (Table 2). The most common indication for surgery was thoracic disc herniation $(23 / 65,35.4 \%)$, which most frequently manifested as myelopathy $(20 / 23,87 \%)$. Three patients with thoracic disc herniation were treated for radiculopathy. All patients with thoracic disc herniation were treated at a single level, except for 1 patient who underwent a 2-level discectomy. This patient presented with myelopathy and was found to have adjacent levels of spinal cord compression. As Table 2 demonstrates, this approach was also performed for treatment of patients with myelopathy caused by tumor $(19 / 65,29.2 \%)$, trauma $(19 / 65,29.2 \%)$, infection $(3 / 65,4.6 \%)$, and deformity $(1 / 65$, $1.5 \%)$.

\section{Clinical Outcomes}

Initially, 2 patients were neurologically worse following lateral extracavitary procedures. One patient with a large calcified disc herniation causing thoracic myelopa- 


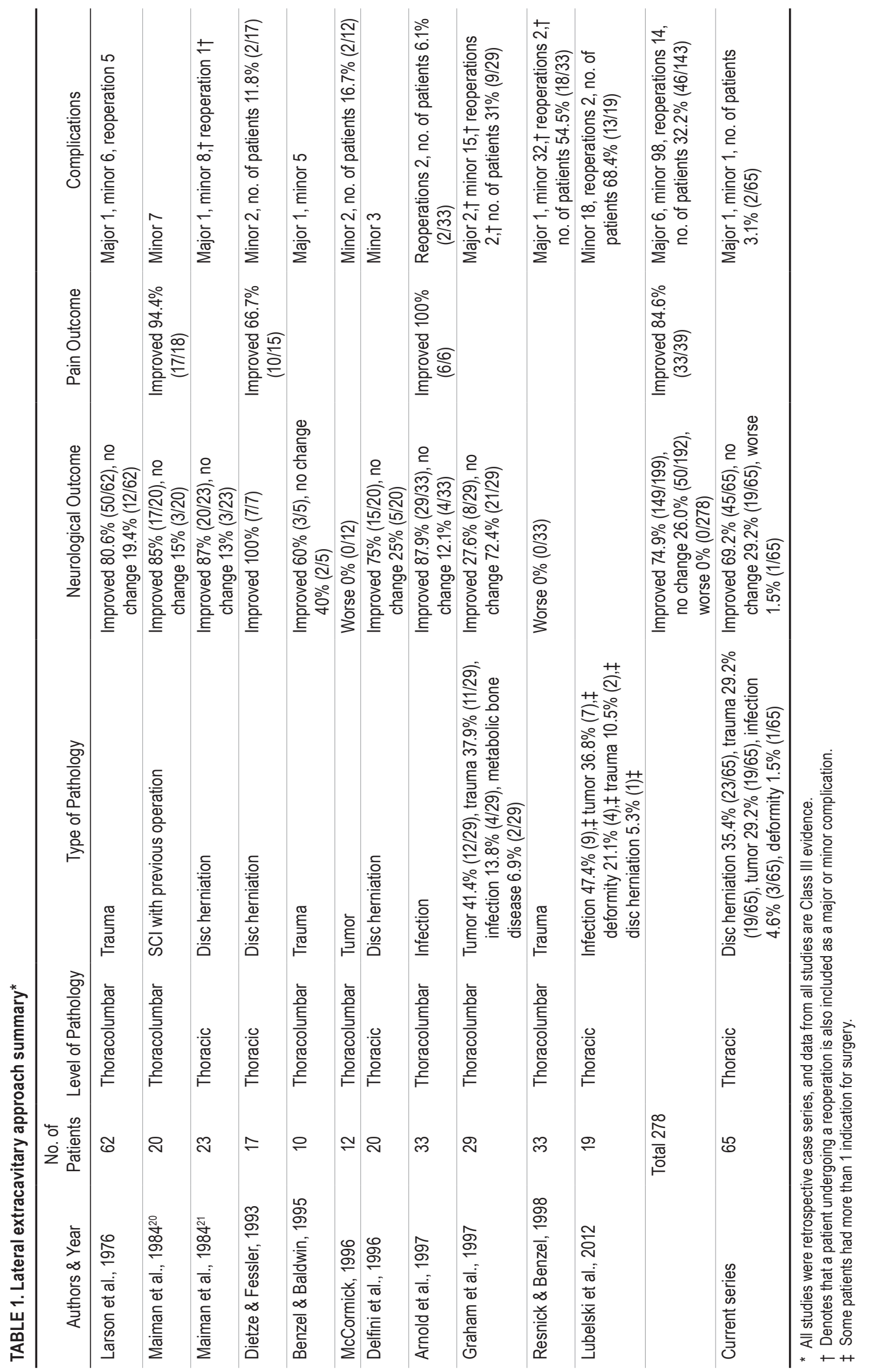


TABLE 2. Surgical indications in 65 patients undergoing the lateral extracavitary approach

\begin{tabular}{lc}
\hline \multicolumn{1}{c}{ Indication } & No. of Patients \\
\hline Thoracic disc herniation & 20 \\
\hline Myelopathy & 3 \\
\hline$\quad$ Radiculopathy & 19 \\
\hline Trauma & \\
\hline$\quad$ Fracture causing spinal cord injury & 6 \\
\hline Tumor & 6 \\
\hline$\quad$ Intradural (meningioma and schwannoma) & 7 \\
\hline Dumbbell & 3 \\
\hline Vertebral body metastasis & 1 \\
\hline Other & \\
\hline Infection & \\
\hline Deformity (hemivertebrae) &
\end{tabular}

thy with paraparesis developed permanently worse left leg function and motor skills following surgery (Fig. 3). In addition to the calcified disc excision, her dura was calcified, necessitating dural excision with patch repair and lumbar drainage for 3 days postoperatively. At 11 years after the operation, she remained ambulatory with an assistance device (cane or walker), had a functional bladder, was able to drive an automobile, but had persistent spasticity in her left lower extremity. The second patient presented with an ASIA Grade C spinal cord injury from a T-10 level burst fracture causing right-sided spinal cord compression. Postoperatively, this patient developed a transient, worse paraparesis (ASIA Grade B); however, over the following months, he improved to ASIA Grade D, and at the 5-year follow-up he was ambulatory with intact bladder and bowel function. At last follow-up, 45 patients (69.2\%) were improved compared with their preoperative neurological status, 19 patients $(29.2 \%)$ were unchanged/stable, and 1 $(1.5 \%)$ was worse over a median follow-up of 48 months (Tables 3 and 4).

On follow-up imaging, all patients with intradural, extramedullary tumors demonstrated gross-total resection and experienced no tumor recurrence at last follow-up (median 4 years). In the 19 trauma patients who underwent the LECA for decompression, internal fixation, and fusion, no patient experienced hardware failure or graft subsidence at last follow-up (median 48 months). One patient treated for myelopathy caused by a left-sided thoracic disc herniation at the T5-6 level developed late rotatory kyphosis. At the 3 -year follow-up evaluation, the deformity was not present; however, at the 10-year follow-up it was radiographically apparent. This change in spinal alignment was stable on dynamic imaging and has not progressed over time, and to date has not required operative treatment.

\section{Complications}

Postoperatively, 1 patient required a chest tube for a pleural rent and resulting pneumothorax and another, detailed above, permanently suffered worse left leg function and spasticity. Of the 6 patients who required intradural dissection for tumor excision and the single patient who
TABLE 3. Outcomes in 46 patients who underwent the LECA for nontraumatic surgical indications

\begin{tabular}{|c|c|}
\hline Surgical Indications & No. of Patients \\
\hline \multicolumn{2}{|c|}{ Thoracic myelopathy from disc herniation } \\
\hline Improved & 19 \\
\hline No change (stable) & 0 \\
\hline Worse & 1 \\
\hline \multicolumn{2}{|c|}{ Thoracic radiculopathy from disc herniation } \\
\hline Improved & 3 \\
\hline No change (stable) & 0 \\
\hline Worse & 0 \\
\hline \multicolumn{2}{|c|}{ Thoracic myelopathy from intradural tumor } \\
\hline Improved & 6 \\
\hline No change (stable) & 0 \\
\hline Worse & 0 \\
\hline \multicolumn{2}{|c|}{ Thoracic myelopathy from dumbbell tumor } \\
\hline Improved & 6 \\
\hline No change (stable) & 0 \\
\hline Worse & 0 \\
\hline \multicolumn{2}{|c|}{$\begin{array}{l}\text { Thoracic pain/myelopathy from metastatic vertebral } \\
\text { column tumor }\end{array}$} \\
\hline Improved & 4 \\
\hline No change (stable) & 3 \\
\hline Worse & 0 \\
\hline \multicolumn{2}{|l|}{ Osteomyelitis/epidural abscess } \\
\hline Improved & 2 \\
\hline No change (stable) & 1 \\
\hline Worse & 0 \\
\hline \multicolumn{2}{|l|}{ Thoracic hemivertebrae with deformity } \\
\hline Improved & 0 \\
\hline No change (stable) & 1 \\
\hline Worse & 0 \\
\hline
\end{tabular}

required dural patch repair, no patient experienced a postoperative CSF leak. Four patients treated for metastatic disease of the thoracic spine died of progression of their primary neoplastic disease: 1 patient at 10 months, 1 at 12 months, 1 at 15 months, and 1 at 17 months postoperatively.

\section{Systematic Review}

The initial PubMed inquiry yielded 62 studies (Fig. 5). Following screening of titles and abstracts, 45 studies were eliminated and 17 were chosen for full-text review. The most common reason for exclusion of full-text articles was failure to report complications and/or outcomes specific to the LECA. After citation review, an additional article was included. The 11 articles that met inclusion criteria are summarized in Table 1. Publication dates ranged from 1976 to 2012. All manuscripts were retrospective case series and considered Class III data. A total of 278 patients were identified. Neurological outcomes were improved in $74.9 \%(149 / 199)$, there was no change in 26\% (50/192), and they were worse in $0 \%(0 / 278)$. Pain outcome was im- 
TABLE 4. Outcomes in 19 patients who underwent the LECA for traumatic surgical indications

\begin{tabular}{ccc}
\hline $\begin{array}{c}\text { Preop ASIA Scale } \\
\text { Grade }\end{array}$ & $\begin{array}{c}\text { Improved/No Change/ } \\
\text { Worse }\end{array}$ & $\begin{array}{c}\text { Postop ASIA Scale } \\
\text { Grade }\end{array}$ \\
\hline A $(n=7)$ & $1 / 6 / 0$ & D/A/NA \\
\hline$B(n=3)$ & $1 / 2 / 0$ & D/B/NA \\
\hline C $(n=3)$ & $2 * / 1 / 0$ & $D, D / C / N A$ \\
\hline$D(n=3)$ & $1 / 2 / 0$ & E/D/NA \\
\hline$E(n=3)$ & $0 / 3+/ 0$ & NA/E/NA \\
\hline
\end{tabular}

NA $=$ not applicable.

* One patient initially experienced worsening to ASIA Grade B but later improved to ASIA Grade D.

$\dagger$ One patient was operated on for radicular pain. This symptom improved postoperatively.

proved $84.6 \%$ (33/39). Outcome reporting varied among articles, which accounts for the different denominators in each group. There were 6 major and 98 minor complications, and 14 reoperations. The percentage of patients suffering a complication was $32.2 \%$ (46/143). The percentage of patients suffering a complication was obtained only from papers reporting the total number of patients suffering a complication, rather than simply totaling the number of complications. This was done to account for the possibility of multiple complications occurring in a single patient.

\section{Discussion}

\section{History}

The origin of the lateral extracavitary approach to the spine dates back to 1894 with the work of Menard..$^{17,23}$ Disenchanted with the operative results of laminectomy for the treatment of Pott's disease, Menard developed what is now known as the "costotransversectomy" approach in an effort to improve lateral access to the spinal column for drainage of paraspinal tubercular abscesses. Despite im- proved access for abscess drainage, outcomes were poor due to lack of antituberculosis chemotherapy and incomplete abscess excision. The early 20th century welcomed a more aggressive surgical approach for the treatment of Pott's disease with a goal of complete abscess removal. Capener, building on the work of Menard, developed the "lateral rachotomy" approach, which transversely divided the erector spinae muscles, improving ventrolateral access to the spine, thus facilitating resection of a ventral mass. ${ }^{5,27}$ Shortly after the original report of the "lateral rachotomy" in 1935, the emergence of antituberculosis chemotherapy forever changed the treatment of Pott's disease.

Sanford Larson and his team at the Medical College of Wisconsin transformed and popularized Capener's lateral approach to the spine in the 1970s and 1980s. ${ }^{16,17}$ Rather than divide the paraspinal musculature, Larson mobilized the musculature, then elevated and retracted it medially. ${ }^{16}$ This novel approach, appropriately named the lateral extracavitary approach, allowed for ventrolateral and posterior access to the spinal column through a single incision. Using the LECA, the surgeon can decompress the ventral and dorsal aspects of the spinal canal while maintaining the ability to perform posterior spinal instrumentation and fusion if necessary.

\section{Technical Advantages}

The LECA is an established option for approaching a wide variety of ventral and ventrolateral pathologies affecting the thoracolumbar spine. This includes disc herniation, tumor (intradural, dumbbell, and metastatic), infection, deformity, and trauma. It is unique in its ability to allow ventral decompression in addition to posterior instrumentation through a single incision.

Traditional anterior approaches via thoracotomy, thorascopic surgery, or sternotomy offer direct anterior or anterolateral exposure of the spinal canal to visualize and access pathology ventral to the thoracic spinal cord with minimal spinal cord manipulation. However, these ap-

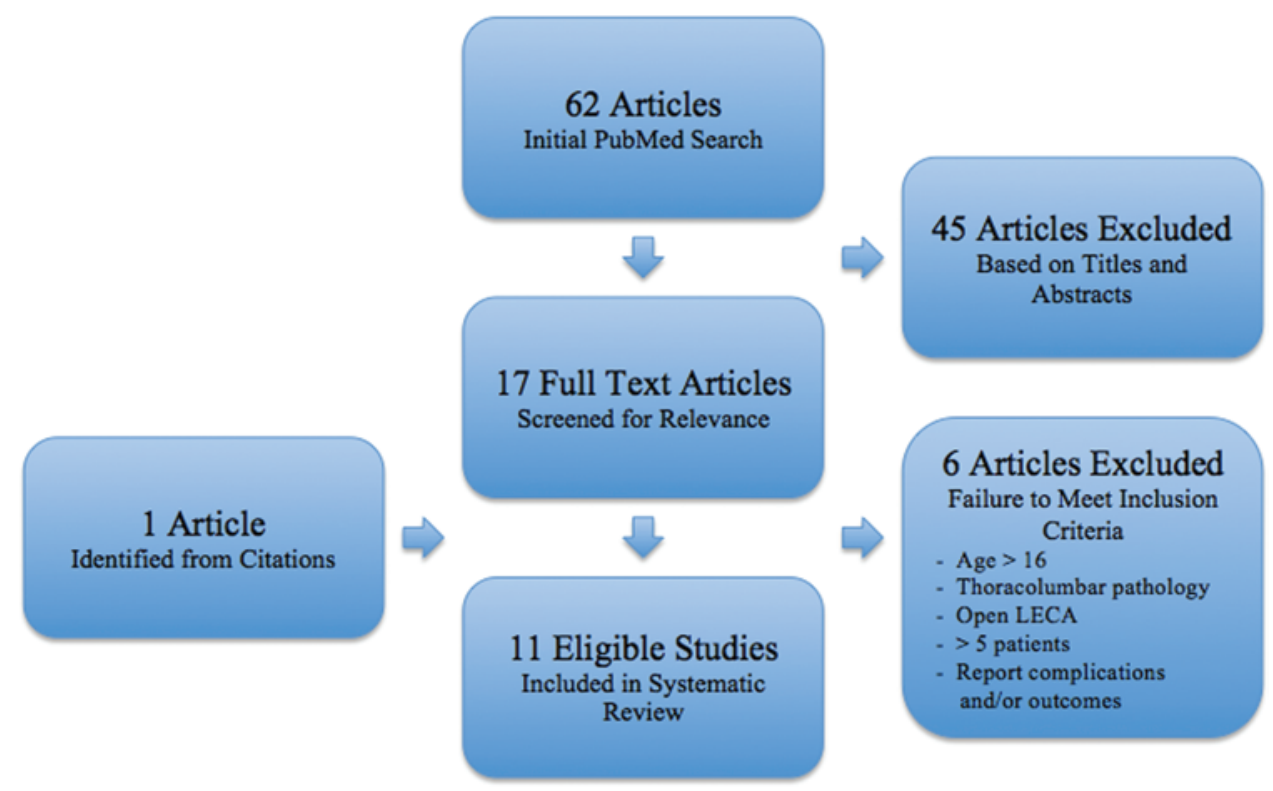

FIG. 5. Flow chart showing the systematic review of the literature. Figure is available in color online only. 
proaches are limited by the morbidity of traversing the thorax, which includes potential lung retraction injury and routine use of chest tubes. Additionally, microsurgery can be technically demanding across the distance of the thorax, particularly in stout patients, and posterior instrumentation is not possible without an additional dorsal operative procedure.

Posterior approaches via laminectomy, transpedicular, or costotransversectomy provide a less morbid approach to the thoracic spine but visualization of pathology ventral to the thoracic spinal cord is limited, often necessitating spinal cord retraction or manipulation. While some authors have reported using a direct posterior approach for treatment of ventral thoracic pathology, ${ }^{31}$ most authors recommend avoiding it, as this technique risks damage to the spinal cord. 2,18,25,29,30 The costotransversectomy approach provides access to ipsilateral ventrolateral pathology extending to, but not across, the midline. ${ }^{19}$ The distinct advantage of the LECA is that the more lateral trajectory allows for visualization ventral to the thoracic spinal cord and across the anatomical midline. This more lateral trajectory also allows for anterior column reconstruction without spinal cord manipulation.

Advancements in minimally invasive spine surgery have provided corridors for the treatment of ventral and ventrolateral pathology of the thoracolumbar spine. $7,12,15,28$ While these approaches allow for smaller incisions with less tissue disruption, ${ }^{11,28}$ they require a unique skill set and fail to provide the operative exposure afforded by an open approach. As minimally invasive approaches continue to evolve, the LECA represents a vetted method for accessing the ventral thoracolumbar spine.

\section{Technical Nuances}

As mentioned in the Operative Technique section above, a bolstered Wilson frame with tilting of the operating table is used to optimize both fluoroscopy and the surgeon's view. While others have reported good results with the 3-quarter prone position, ${ }^{3}$ they experienced trouble with radiographic localization and a position-related peripheral nerve palsy. The bolstered Wilson frame elevates the patient off the bed allowing for acquisition of true anteroposterior and lateral radiographs for localization and instrumentation. No position-related complications were encountered in the current series.

To maximize the lateral trajectory of the LECA, the latissimus bundle should be elevated and the patient rotated away from the surgeon so that direct visualization of the contralateral aspects of the lesion is achieved. This is key when addressing pathology ventral to the thoracic spinal cord that crosses the midline. Telfa (Covidien) dressing can be placed on the pleura and a wide-based malleable retractor used for gentle ventral retraction of the pleura and underlying lung during key points in the operation. These maneuvers will provide crucial millimeters of additional space for dissection and drilling in narrow corridors.

Given the unavoidable damage to segmental arterial branches that may supply the thoracic spinal cord, preoperative spinal angiography has been recommended by some authors in an effort to identify the artery of Adamkiewicz. ${ }^{6}$ We do not typically obtain spinal angiograms prior to performing the LECA. Spinal angiography is associated with risks, including access site complications and distal embolic phenomena ${ }^{13}$ and is believed to provide no benefit in this setting. Despite the theoretical risk of spinal cord ischemia due to interruption of a segmental radicular artery, we have not encountered a single incident of an iatrogenic vascular ischemic injury to the thoracic spinal cord.

Optimizing surgical outcomes in spinal cord surgery involves more than technical expertise. The surgeon must also communicate the importance of avoidance of hypotension to his or her anesthesia and critical care team. We insist on maintenance of a mean arterial pressure of 85 $\mathrm{mm} \mathrm{Hg}$ throughout surgery and in the immediate postoperative period. This strategy was implemented in all 65 cases and is felt to be an integral component of these procedures to optimize the potential of a good neurological outcome.

\section{Patient Selection}

The selection of patients best suited for the LECA hinges on 3 salient features: 1) location of pathology below T-2 (i.e., T-3 and below); 2) the need for ventral decompression of the spinal cord; and 3) the potential need for anterior intervertebral reconstruction with or without posterior instrumentation and fusion. While transpedicular and costotransversectomy approaches have gained popularity due to their technical ease and relatively low morbidity, they do not typically allow for ventral decompression across the midline or anterior column reconstruction. The senior author (M.N.H.) continues to use the LECA for select cases meeting the aforementioned criteria with excellent results. Limitations of the approach must also be appreciated. Lateral extracavitary access at or above the level of T-3 is technically challenging due to the upper thoracic curvature and the presence of the scapula, which limits a more lateral exposure and trajectory.

\section{Outcomes}

A major advantage of the LECA is its ability to provide direct visualization and access to ventrolateral thoracolumbar spinal pathology without spinal cord manipulation. This technical advantage has led to excellent neurological outcomes with nearly $75 \%$ of patients described in the literature demonstrating neurological improvement. $1,8,9,16,20,21$ Furthermore, Maiman et al. reported significant neurological improvement following late anterior decompression with restoration of a normal anatomical relationship of the spinal cord and spinal canal using the LECA in patients previously treated with laminectomy or spinal instrumentation, or both..$^{20}$ Pain associated with thoracolumbar pathology also tends to respond well to the LECA, with approximately $85 \%$ of patients experiencing meaningful relief. $1,9,20$

While our systematic review failed to identify a single case of neurological worsening reported in the literature, the current case series encountered 1 such complication. This complication arose following excision of a large calcified thoracic disc and left the patient functional and ambulatory but with permanently worse left leg spasticity. 
Her injury is believed to be the result of dissection of the large calcified disc and involved dura from the left ventrolateral thoracic spinal cord despite adequate visualization and access. This risk is inherent to large calcified discs and would not likely have been mitigated by an alternative operative approach.

\section{Complications}

Historically, the reporting of complications associated with the LECA has varied widely leading to large variations in the incidence of complications. More recent series report complication rates of $31 \%$ to $68 \% .^{14,19,26}$ In addition to expected surgical complications (i.e., wound infection, DVT, urinary tract infection), the LECA is uniquely prone to pulmonary complications compared with traditional posterior approaches. The risk of pleural rents resulting in pneumothorax, pleural effusion, or both, necessitating the placement of a chest tube should be considered in preoperative planning, especially in a patient with preexisting pulmonary dysfunction.

\section{Limitations}

The documented case series is limited by its retrospective nature and the participation of a single surgeon. Retrospective data collection is subject to bias in both the original and subsequent reporting of information, in addition to incomplete data sets. Reporting of a single senior surgeon's experience raises concern about the generalizability of the results.

The systematic review is limited by the quality of the original articles. Publication dates range from 1976 to 2012. All 11 series were retrospective case series and considered Class III evidence. Included series encompass a wide variety of data reporting methods. Most series, including the present, used a nonvalidated outcome scale of "improved," "no change," and "worse" for patients treated for thoracic myelopathy, rather than more contemporary assessments (i.e., modified Japanese Orthopaedic Association [JOA]). The current series was initiated in 1993 and used the standard outcome schemes for that time, specifically the ASIA scale for traumatic spinal cord injury and the 3-tiered "improved, no change, and worse" scale for nontraumatic pathology. Only over the last 8 years has the senior author (M.N.H.) used the modified JOA score to quantify a patient's preoperative and postoperative neurological status, a scheme that could not be applied to the entire study population. Additionally, each article had a topic of emphasis that influenced the reporting of end points.

\section{Conclusions}

The lateral extracavitary approach provides ventrolateral access to the thoracolumbar spine and spinal cord while allowing for posterior instrumentation through the same incision. This technique permits ventral decompression of the spinal cord well across the midline and anterior reconstruction of the spinal column, with the potential for dorsal internal fixation and fusion. While excellent neurological results can be expected, the risk of pulmonary complications should be considered during patient selection and counseling.

\section{References}

1. Arnold PM, Baek PN, Bernardi RJ, Luck EA, Larson SJ: Surgical management of nontuberculous thoracic and lumbar vertebral osteomyelitis: report of 33 cases. Surg Neurol 47:551-561, 1997

2. Benson MK, Byrnes DP: The clinical syndromes and surgical treatment of thoracic intervertebral disc prolapse. J Bone Joint Surg Br 57:471-477, 1975

3. Benzel EC: The lateral extracavitary approach to the spine using the three-quarter prone position. J Neurosurg 71:837841,1989

4. Benzel EC, Baldwin NG: Crossed-screw fixation of the unstable thoracic and lumbar spine. J Neurosurg 82:11-16, 1995

5. Capener N: The evolution of lateral rhachotomy. J Bone Joint Surg Br 36-B:173-179, 1954

6. Champlin AM, Rael J, Benzel EC, Kesterson L, King JN, Orrison WW, et al: Preoperative spinal angiography for lateral extracavitary approach to thoracic and lumbar spine. AJNR Am J Neuroradiol 15:73-77, 1994

7. Dakwar E, Smith WD, Malone KT, Uribe JS: Minimally invasive lateral extracavitary resection of foraminal neurofibromas. J Clin Neurosci 18:1510-1512, 2011

8. Delfini R, Di Lorenzo N, Ciappetta P, Bristot R, Cantore G: Surgical treatment of thoracic disc herniation: a reappraisal of Larson's lateral extracavitary approach. Surg Neurol 45:517-523, 1996

9. Dietze DD Jr, Fessler RG: Thoracic disc herniations. Neurosurg Clin N Am 4:75-90, 1993

10. Ditunno JF Jr, Young W, Donovan WH, Creasey G: The international standards booklet for neurological and functional classification of spinal cord injury. Paraplegia 32:70-80, 1994

11. Eichholz KM, O’Toole JE, Fessler RG: Thoracic microendoscopic discectomy. Neurosurg Clin N Am 17:441-446, 2006

12. Falavigna A, Piccoli Conzatti L: Minimally invasive approaches for thoracic decompression from discectomy to corpectomy. J Neurosurg Sci 57:175-192, 2013

13. Forbes G, Nichols DA, Jack CR Jr, Ilstrup DM, Kispert DB, Piepgras DG, et al: Complications of spinal cord arteriography: prospective assessment of risk for diagnostic procedures. Radiology 169:479-484, 1988

14. Graham AW III, Mac Millan M, Fessler RG: Lateral extracavitary approach to the thoracic and thoracolumbar spine. Orthopedics 20:605-610, 1997

15. Khoo LT, Smith ZA, Asgarzadie F, Barlas Y, Armin SS, Tashjian V, et al: Minimally invasive extracavitary approach for thoracic discectomy and interbody fusion: 1-year clinical and radiographic outcomes in 13 patients compared with a cohort of traditional anterior transthoracic approaches. J Neurosurg Spine 14:250-260, 2011

16. Larson SJ, Holst RA, Hemmy DC, Sances A Jr: Lateral extracavitary approach to traumatic lesions of the thoracic and lumbar spine. J Neurosurg 45:628-637, 1976

17. Lifshutz J, Lidar Z, Maiman D: Evolution of the lateral extracavitary approach to the spine. Neurosurg Focus 16(1):E12, 2004

18. Logue V: Thoracic intervertebral disc prolapse with spinal cord compression. J Neurol Neurosurg Psychiatry 15:227241, 1952

19. Lubelski D, Abdullah KG, Mroz TE, Shin JH, Alvin MD, Benzel EC, et al: Lateral extracavitary vs. costotransversectomy approaches to the thoracic spine: reflections on lessons learned. Neurosurgery 71:1096-1102, 2012

20. Maiman DJ, Larson SJ, Benzel EC: Neurological improvement associated with late decompression of the thoracolumbar spinal cord. Neurosurgery 14:302-307, 1984

21. Maiman DJ, Larson SJ, Luck E, El-Ghatit A: Lateral extra- 
cavitary approach to the spine for thoracic disc herniation: report of 23 cases. Neurosurgery 14:178-182, 1984

22. McCormick PC: Surgical management of dumbbell and paraspinal tumors of the thoracic and lumbar spine. Neurosurgery 38:67-75, 1996

23. Ménard V: Causes de la paraplegia dans le mal de Pott. Son traitement chirurgical par l'ouverture direct du foyer tuberculeux des vertebras. Rev Orthop 5:47-64, 1894

24. Moher D, Liberati A, Tetzlaff J, Altman DG: Preferred reporting items for systematic reviews and meta-analyses: the PRISMA statement. Open Med 3:e123-130, 2009

25. Morgan TH, Wharton GW, Austin GN: The results of laminectomy in patients with incomplete spinal cord injuries. Paraplegia 9:14-23, 1971

26. Resnick DK, Benzel EC: Lateral extracavitary approach for thoracic and thoracolumbar spine trauma: operative complications. Neurosurgery 43:796-803, 1998

27. Seddon HJ: Pott's paraplegia: prognosis and treatment. Br J Surg 22:769-799, 1935

28. Snyder LA, Smith ZA, Dahdaleh NS, Fessler RG: Minimally invasive treatment of thoracic disc herniations. Neurosurg Clin N Am 25:271-277, 2014

29. Solero CL, Fornari M, Giombini S, Lasio G, Oliveri G, Cimino C, et al: Spinal meningiomas: review of 174 operated cases. Neurosurgery 25:153-160, 1989

30. Steck JC, Dietze DD, Fessler RG: Posterolateral approach to intradural extramedullary thoracic tumors. J Neurosurg 81:202-205, 1994
31. Voulgaris S, Alexiou GA, Mihos E, Karagiorgiadis D, Zigouris A, Fotakopoulos G, et al: Posterior approach to ventrally located spinal meningiomas. Eur Spine J 19:11951199,2010

\section{Disclosures}

The authors report no conflict of interest concerning the materials or methods used in this study or the findings specified in this paper.

\section{Author Contributions}

Conception and design: Foreman, Moore, Hadley. Acquisition of data: Foreman, Naftel, Hadley. Analysis and interpretation of data: Foreman, Hadley. Drafting the article: Foreman, Naftel. Critically revising the article: Foreman, Moore, Hadley. Reviewed submitted version of manuscript: Foreman, Hadley. Approved the final version of the manuscript on behalf of all authors: Foreman. Study supervision: Moore, Hadley.

\section{Correspondence}

Paul M. Foreman, Department of Neurosurgery, University of Alabama at Birmingham, 510 20th St. S, FOT 1062 ,

Birmingham, AL 35294-3410. email: pforeman03@gmail.com. 